Check for updates

Cite this: RSC Adv., 2019, 9, 32601

Received 18th August 2019

Accepted 3rd October 2019

DOI: $10.1039 / c 9 r a 06452 d$

rsc.li/rsc-advances

\section{A novel AIE-active dye for fluorescent nanoparticles by one-pot combination of Hantzsch reaction and RAFT polymerization: synthesis, molecular structure and application in cell imaging ${ }^{\dagger}$}

\author{
Yali Chen, ${ }^{\text {ab }}$ Zengfang Huang, (D)*ab Xiaobo Liu, ${ }^{b}$ Liucheng Mao, ${ }^{c}$ Jinying Yuan, (D) \\ Xiaoyong Zhang, (D) ${ }^{\mathrm{d}}$ Lei Tao (D) ${ }^{\mathrm{c}}$ and Yen Weic
}

In recent years, amphiphilic AlE-active fluorescent organic materials with aggregation-induced emission (AIE) properties have been extensively investigated due to their excellent properties. This study describes the synthesis of a novel AIE-active dye of tetraphenylethylene diphenylaldehyde (TPDA). As compared with the reported fluorescent dye TPB, the fluorescence intensity of TPDA is significantly enhanced with the distinct red shift of emission wavelength. Subsequently, the corresponding novel polymers PEG-TPD were obtained through the one-pot combination of Hantzsch reaction and RAFT polymerization. The structure of PEG-TPD1 by the two-step process was similar with that of PEG-TPD2 by the one-pot method at the same feeding ratio of TPDA and PEGMA. The molecular weights $\left(M_{n}\right)$ of the polymers PEG-TPD1 and PEG-TPD2 were respectively 52000 and 28000 with narrow polydispersity index (PDI), and their molar fractions of TPDA were respectively about $9.5 \%$ and $14.3 \%$, indicating that the degree of Hantzsch reaction in the one-pot process was more complete. Subsequently, the effect of feed ratio of TPDA and PEGMA on polymer structure was further studied. It can be seen that the $M_{n}$ of the polymers gradually increases as the proportion of TPDA increases. In aqueous solution, these amphiphilic PEGTPD polymers tended to self-assemble into corresponding fluorescent polymer nanoparticles (FPNs). The diameter of PEG-TPD2 FPNs ranged from 200 to $300 \mathrm{~nm}$, and their fluorescence emission spectra have maximum emission peak at $509 \mathrm{~nm}$. The PEG-TPD FPNs have significant advantages such as good fluorescence intensity, high water dispersibility, good biocompatibility and easy absorption by cells, which can be attractively used in the field of bioimaging.

\section{Introduction}

Biological systems are one of the most precise and complex systems. As a direct-viewing and visual biological imaging technology, fluorescence imaging has become one of the most effective tools to help us understand the key information of various physiological and pathological processes in biological systems. ${ }^{1-5}$ Currently, many different types of fluorescent

${ }^{a}$ School of Materials \& Food Engineering, Zhongshan Institute, University of Electronic Science \& Technology of China, Zhongshan, 528402, P. R. China. E-mail: hzf105@163. com

${ }^{b}$ School of Materials and Energy, University of Electronic Science \& Technology of China, Chengdu, 610054, P. R. China

'Department of Chemistry, The Tsinghua Center for Frontier Polymer Research, Tsinghua University, Beijing 100084, P. R. China

${ }^{d}$ Department of Chemistry, Nanchang University, Nanchang 330047, P. R. China $\dagger$ Electronic supplementary information (ESI) available. CCDC 1893700. For ESI and crystallographic data in CIF or other electronic format see DOI: 10.1039/c9ra06452d materials have been reported, which can be mainly divided into two major categories, namely fluorescent inorganic materials and fluorescent organic materials. Fluorescent inorganic materials can be further divided into semiconductor quantum dots, carbon dots and so on. Fluorescent organic materials can be further divided into organic small molecular dyes, fluorescent polymer nanoparticles (FPNs), etc. ${ }^{6-11}$ Fluorescent inorganic materials have the advantage of low cost, convenient preparation, good light stability and ideal properties, but they also have significant disadvantages: they will accumulate in organisms due to their difficulty in degradation, and the metal ions from them are toxic to organisms. ${ }^{12}$ In contrast, FPNs have better design, biodegradability and lower toxicity. However, many traditional FPNs also have one obvious disadvantage: the aggregation quenching effect (ACQ), which makes the dyes emit the strong fluorescence in good solution, while the luminescence is very weak or not in the aggregate state. Tang's group firstly reported the phenomenon of aggregation-induced emission (AIE) which makes the dyes have very weak luminescent or 
not luminescent in good solution, but their fluorescence intensity in the aggregate state is obviously enhanced. ${ }^{13}$ Since then, more and more researchers have focused on the development of novel AIE-active molecules, and some typical AIEactive dyes have been developed such as tetraphenylethene (TPE), hexaphenylsilole (HPS) and distyreneanthracene (DSA) derivatives. ${ }^{\text {14-20 }}$ However, AIE-active molecules have inherent hydrophobicity due to their structure and are difficult to be directly applied in fluorescence imaging. Therefore, in order to solve this problem, AIE-active molecules are usually converted into amphiphilic AIE-active fluorescent nanoparticles. A series of preparation strategies for fluorescent nanoparticles have been developed such as polymer physical encapsulation, acid anhydride ring-opening polymerization, Schiff base condensation, free radical polymerization, controlled living polymerization, etc. ${ }^{21-31}$ In a previous report, AIE-active FPNs by one-pot Mannich reaction were described. The AIE-active dye PhE-OH was subjected to Mannich reaction with paraformaldehyde and 4-arm PEG-NH $\mathrm{H}_{2}$ to obtain PEG-PhE FPNs, showing that PEG-PhE FPNs had potential applications in the bioimaging field. ${ }^{32}$ Novel aldehyde-functionalized AIE-active tetraphenylethene derivatives have been synthesized for indirect detection of organophosphorus pesticides with a low detection limit of 0.008 $\mathrm{mg} \mathrm{L}^{-1}$ and a broad detection range of $0.009-22.5 \mathrm{mg} \mathrm{L}^{-1}$, providing a new strategy for detecting organophosphorus pesticides in the agriculture and environment. ${ }^{33}$

As an active controlled radical polymerization technique, reversible addition-fragmentation chain transfer (RAFT) polymerization has developed in recent years, which has been an effective way to obtain amphiphilic polymers with controllable molecular weight and narrow PDI. ${ }^{34,35}$ Multicomponent reaction (MCRs) is a unique synthetic method in which three or more reactants are added to a reactor for simultaneous chemical reactions. Without separation of intermediates, complex molecules containing structural fragments of all the reactants are obtained directly. Many researchers have conducted extensive investigations on MCRs including Hantzsch reaction, Biginelli reaction, Passerini reaction, Ugi reaction and so on. Among them, The Hantzsch reaction is one of the most effective and classical methods for the synthesis of 1,4-dihydropyridine derivatives, which has the advantages of mild reaction conditions, high atomic economy, etc. With the rapid development of polymer science, the exploration of various MCRs potentials has been successfully carried out for the synthesis and application of multifunctional polymer materials. ${ }^{36}$ An ingenious one-pot method for the synthesis of AIE-active FPNs has been reported. The hydrophobic AIE-active dye 4-(1,2,2-triphenylvinyl) benzaldehyde (TPB) and hydrophilic monomer PEGMA were successfully used to prepare amphiphilic AIE-active FPNs via the combination of RAFT polymerization and Hantzsch reaction, providing a simple and efficient way to prepare multifunctional AIE-based fluorescent materials. ${ }^{37}$ Inspired by the efficient use of limited amino acids to synthesize large amounts of protein in organisms, a two-stage method for the synthesis of polymers has been investigated. Six main-chain structure controlled polymer precursors were rapidly prepared by ultrafast RAFT polymerization, and then these polymer precursors were modified by Hantzsch reaction to obtain polymers with 1,4-dihydropyridine as side chain, which indicated that Hantzsch reaction offered a new and efficient option for the preparation of polymers with pendant 1,4-dihydropyridine groups. ${ }^{38}$ The Hantzsch reaction has been used for the detection of formaldehyde for decades due to its fast reaction rate and natural fluorescence property of 1,4-dihydropyridine. A direct method for the detection of formaldehyde in living organisms by the $\beta$-ketone-ester-containing polymer fluorescent probe via Hantzsch reaction has been reported, which exhibited sensitivity to the detection of formaldehyde and good biocompatibility. ${ }^{39}$

Owing to the high atomic economy of Hantzsch reaction and the good adjustable effect, mild conditions of RAFT polymerization, one-pot combination of RAFT polymerization and Hantzsch reaction was adopted to conveniently synthesize novel AIE-active polymers. In this contribution, a novel AIE-active dye TPDA with terminal aldehyde group was synthesized for the first time. As compared with the reported TPB dye, the fluorescence intensity of TPDA is significantly enhanced with the obvious red shift of emission wavelength. Then the corresponding new AIEactive polymers PEG-TPD were prepared by one-pot combination of RAFT polymerization and Hantzsch reaction taking TPDA and PEGMA as hydrophobic and hydrophilic segments. In aqueous solution, the amphiphilic polymers PEG-TPD2 tended to self-assemble into core-shell FPNs with 200-300 nm diameters, and their fluorescence emission spectra have maximum emission peak at $509 \mathrm{~nm}$ with the obvious AIE phenomenon. Subsequently, the cytotoxicity and cell uptake behaviour of PEG-TPD2 FPNs were studied in detail. The results showed the great potential of PEG-TPD FPNs in the field of bioimaging due to their various excellent properties such as good fluorescence intensity, high water dispersibility and good biocompatibility.

\section{Experimental}

\subsection{Materials and characterization}

Tetrabutylammonium bromide (TBAB) (Aladdin, 99\%), 4-formylphenylboronic acid (Aladdin, 97\%), tetrakis(triphenylphosphine)palladium(0) (Aladdin, $\mathrm{Pd} \geq 8.9 \%$ ), potassium carbonate, 2-(acetoacetyloxy)ethyl methacrylate (AEMA, Aladdin, 95\%), 5,5-dimethyl-1,3-cyclohexadione (Aladdin, 99\%), ammonium acetate, glycine (Aladdin, 99\%), 2,2'-azobis(2-methylpropionitrile) (AIBN, Aladdin, 99\%) and poly(ethylene glycol) methacrylate (PEGMA, $M_{\mathrm{n}}=475$, Aladdin) were purchased from commercial companies and directly used without further treatment. The organic solvents used in the experiments were analytical pure and were directly used without any purification. Tetraphenylethene-functionalized bromophenylacetonitrile (TPEBr) and chain transfer agent of 4-cyano-4(ethylthiocarbonothioylthio) pentanoic acid (CTA) were prepared based on the previously reported methods. ${ }^{\mathbf{4 0 - 4 3}}$

${ }^{1} \mathrm{H}$ NMR spectra of dye TPDA, polymers PEG-AE and PEGTPD were obtained from a JEOL JNM-ECA $400(400 \mathrm{MHz})$ spectrometer and tetramethylsilane was used as a reference. Data on the $M_{\mathrm{n}}$ and PDI of the synthetic polymers were obtained 
by gel permeation chromatography (GPC) using a Shimadzu LC20AD GPC system with a Shimadzu RID-10A refractive index detector, which was calibrated with standard polystyrene before the experiment and used $N, N$-dimethylformamide (DMF) as the eluent at the $1 \mathrm{~mL} \mathrm{~min}^{-1}$ flow rate. FT-IR spectrum of dye TPDA was obtained from a PerkinElmer Spectrum 100 spectrometer (Waltham, MA, USA) by a KBr method while the FT-IR spectra of polymers PEG-AE and PEG-TPD were obtained by a reflection mode. Transmission electron microscopy (TEM) images were obtained using a JEM-1200EX microscope at $100 \mathrm{kV}$ accelerating voltage. A drop of PEG-TPD2 suspension was added to a carboncoated copper grid and then dried naturally to get TEM sample. UV-visible absorption spectra were measured from a PerkinElmer LAMBDA $35 \mathrm{UV} / \mathrm{Vis}$ spectroscopy system. The fluorescence emission spectra were measured using a PELS-55 spectrometer. Single crystal X-ray diffraction data of TPDA dye was acquired from a Bruker Smart CCD diffractometer at $100 \mathrm{~K}$, the structure of which was solved by Patterson methods followed by different Fourier syntheses and then refined by fullmatrix least squares techniques against $\mathrm{F}_{2}$ with SHELXTL. ${ }^{\mathbf{4 4}}$

\subsection{Preparation of tetraphenylethene-functionalized(4- acetonitrile) diphenylaldehyde (TPDA)}

TPEBr (1.08 g, $2.00 \mathrm{mmol}$ ), 4-formylbenzeneboronic acid (0.360 g, $2.40 \mathrm{mmol})$, ТВAB (64.5 $\mathrm{mg}, 0.200 \mathrm{mmol})$, potassium carbonate aqueous solution $\left(2.00 \mathrm{~mol} \mathrm{~L}^{-1}, 3.60 \mathrm{~mL}\right)$ and toluene $(14.0 \mathrm{~mL})$ were added to a Schlenk tube, which was magnetically stirred for $0.5 \mathrm{~h}$ at room temperature. Then tetrakis(triphenylphosphine)palladium(0) $\left(2.70 \mathrm{mg}, 2.34 \times 10^{-3} \mathrm{mmol}\right)$ was added to the Schlenk tube and the mixture was deoxygenated by stirring for another $0.5 \mathrm{~h}$ at room temperature under $\mathrm{N}_{2}$ gas. Then the above reaction mixture reacted at $90{ }^{\circ} \mathrm{C}$ for $48 \mathrm{~h}$ with magnetic stirring. When the reaction was over, the reaction mixture was poured into a separated funnel containing some water and then extracted three times with a small amount of dichloromethane. Then the organic phase was dried for several hours with anhydrous magnesium sulfate and filtered to produce a transparent solution. The solvent was rapidly evaporated by means of a rotary evaporator which was controlled under reduced pressure. Finally, the residue was chromatographed on a silica gel column using $n$-hexanedichloromethane $(\mathrm{v} / \mathrm{v}=2: 1)$ to obtain TPDA. (0.928 g, 82.3\% yield). ${ }^{1} \mathrm{H}$ NMR (400 MHz, $\left.\mathrm{CDCl}_{3}, \delta\right): 7.03-7.07$ (m, 6H; Ar H), 7.03-7.07 (m, 11H; Ar H), 7.49 (s, 1H; CH), 7.68-7.79 (m, 8H; Ar H), 7.97-7.99 (d, $J=8.0 \mathrm{~Hz}, 2 \mathrm{H}$; Ar H), 10.07 (s, 1H; -CHO).

\subsection{Two-step method synthesis of PEG-TPD}

AEMA (42.8 mg, $0.200 \mathrm{mmol}$ ), PEGMA (0.500 g, $1.05 \mathrm{mmol}$ ), AIBN (2.00 mg, $0.0122 \mathrm{mmol})$, CTA (3.1 mg, $0.0120 \mathrm{mmol}$ ) and toluene $(3.00 \mathrm{~mL})$ were added to a Schlenk tube, which was deoxygenated five times in a vacuum-nitrogen cycle after being frozen by liquid nitrogen. After the above reaction mixture was returned to room temperature, it was reacted at $70{ }^{\circ} \mathrm{C}$ for $36 \mathrm{~h}$ with magnetic stirring. The solvent was rapidly evaporated by means of a rotary evaporator which was controlled under reduced pressure. The polymer was precipitated three times from tetrahydrofuran into petroleum ether. Then the obtained pure polymer was dried under vacuum and named as PEG-AE.

TPDA (62.1 $\mathrm{mg}, \quad 0.110 \mathrm{mmol}), \quad$ 5,5-dimethyl-1,3cyclohexadione $(15.4 \mathrm{mg}, 0.110 \mathrm{mmol})$, ammonium acetate (12.7 mg, $0.165 \mathrm{mmol}$ ), glycine (1.70 mg, $0.0220 \mathrm{mmol}$ ), PEG-AE $(0.284 \mathrm{~g})$ and a mixture of acetonitrile $(2.50 \mathrm{~mL})$ and tetrahydrofuran $(1.50 \mathrm{~mL})$ were added into a Schlenk tube, which was deoxygenated five times in a vacuum-nitrogen cycle after being frozen by liquid nitrogen. After the above reaction mixture was returned to room temperature, it was reacted at $70{ }^{\circ} \mathrm{C}$ for $36 \mathrm{~h}$ with magnetic stirring. Then the reaction was dialyzed successively by acetonitrile and acetone for $36 \mathrm{~h}$. The solvent was rapidly evaporated by means of a rotary evaporator which was controlled under reduced pressure. The polymer was precipitated three times from tetrahydrofuran into petroleum ether. Then the obtained pure polymer was dried under vacuum and named as PEG-TPD1.

\subsection{One-pot method synthesis of PEG-TPD}

TPDA (56.3 mg, $0.100 \mathrm{mmol}$ ), 5,5-dimethyl-1,3-cyclohexadione $(14.0 \mathrm{mg}, 0.100 \mathrm{mmol})$, ammonium acetate $(11.6 \mathrm{mg}, 0.150$ $\mathrm{mmol}$ ), glycine (1.10 mg, $0.0150 \mathrm{mmol})$, AEMA $(21.4 \mathrm{mg}, 0.100$ mmol), PEGMA (0.250 g, $0.526 \mathrm{mmol})$, AIBN (1.00 mg, $6.10 \times$ $\left.10^{-3} \mathrm{mmol}\right)$, CTA $\left(1.60 \mathrm{mg}, 6.00 \times 10^{-3} \mathrm{mmol}\right)$ and a mixture of acetonitrile $(1.50 \mathrm{~mL})$ and tetrahydrofuran $(1.50 \mathrm{~mL})$ were added to a Schlenk tube, which was deoxygenated five times in a vacuum-nitrogen cycle after being frozen by liquid nitrogen. After the above reaction mixture was returned to room temperature, it was reacted at $70{ }^{\circ} \mathrm{C}$ for $36 \mathrm{~h}$ with magnetic stirring. Then the reaction was dialyzed successively by acetonitrile and acetone for $36 \mathrm{~h}$. The solvent was rapidly evaporated by means of a rotary evaporator which was controlled under reduced pressure. The polymer was precipitated three times from tetrahydrofuran into petroleum ether. Then the obtained pure polymer was dried under vacuum and named PEG-TPD2. Different polymers were prepared according to the above same method with different ratios of TPDA to PEGMA, and corresponding polymers were respectively named as PEG-TPD3 (with TPDA : PEGMA ratio of 1:4) and PEG-TPD4 (with TPDA : PEGMA ratio of $1: 3$ ).

\section{Results and discussion}

The synthetic route of novel AIE-active dye TPDA was shown in Scheme 1(A), and the synthesis of its amphiphilic fluorescent polymers PEG-TPD by one-pot combination of Hantzsch reaction and RAFT polymerization was shown in Scheme 1(B) with their cell imaging applications. Hantzsch reaction was carried out with TPDA dye, 5,5-dimethyl-1,3-cyclohexadione, ammonium acetate and functional monomer AEMA as reaction reagents to form intermediate monomer TPD-MA. Subsequently, the obtained TPD-MA would in situ polymerize with hydrophilic PEGMA monomer to prepare new AIE-active polymers PEG-TPD. The simultaneous presence of hydrophobic TPDA segment and hydrophilic PEGMA segment induced the amphiphilic fluorescent PEG-TPD polymers to self-assemble 

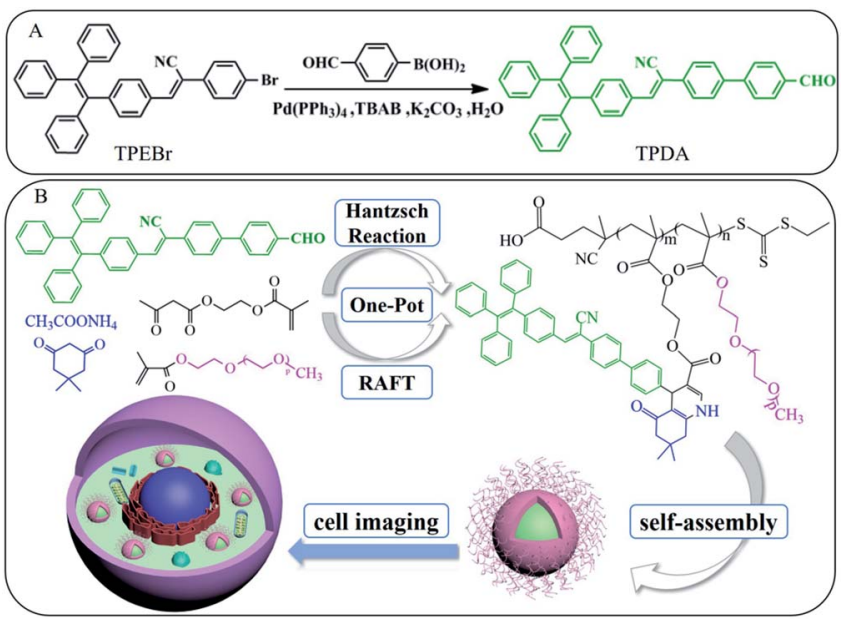

Scheme 1 (A) Synthetic route of TPDA, (B) schematic diagram of PEGTPD polymers by one-pot method and their application in cell imaging.

into PEG-TPD FNPs in water, which appeared as a core-shell structure of hydrophobic segment inside and hydrophilic segment outside and could be internalized by the cells.

The ORTEP diagram of complex TPDA dye indicates that TPDA belongs to triclinic crystal system and $\mathrm{P} \overline{1}$ space group as shown in Fig. 1. $a=5.4879(5) \AA, b=9.4465(18) \AA, c=29.547(2)$ $\AA, \alpha=91.818(10)^{\circ}, \beta=92.824(7)^{\circ}, \gamma=100.708(11)^{\circ}, V=$ $1502.0(3) \AA^{3}, Z=2, R_{1}=0.0878(I>2 \sigma(I))$, and $w R_{2}=0.2189$. The dihedral angle between the benzene rings of C14-C15-C16C17-C18-C19 and C20-C21-C22-C23-C24-C25 is about $74.794^{\circ}$, and the dihedral angle between the benzene ring of C9-C10-C11-C12-C13-C42 and C2-C3-C4-C5-C6-C7 is about $75.108^{\circ}$, indicating the angulation structure of the phenyl rings owing to the steric effect of TPE segment. The $\mathrm{C}=\mathrm{O}$ bond and C35-C36-C37-C38-C39-C40 are almost at the same plane, which endows TPDA dye the good conjugative effect.

To demonstrate the successful synthesis of PEG-TPD, the ${ }^{1} \mathrm{H}$ NMR spectroscopy of TPDA, PEG-AE and PEG-TPD polymers

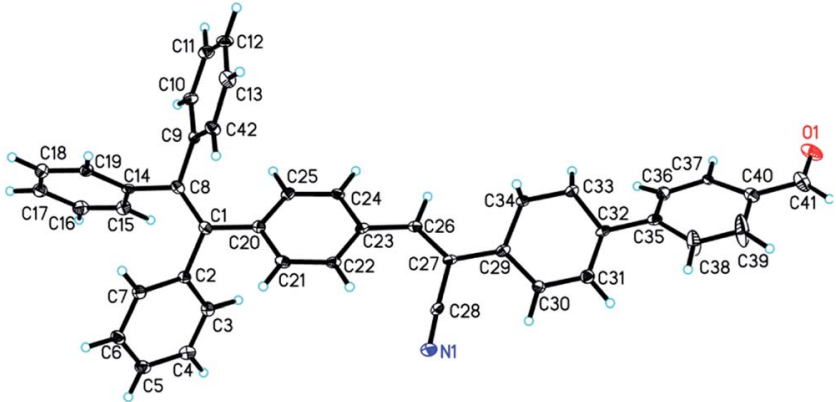

Fig. 1 ORTEP plot of TPDA dye. Selected bond distance $(\AA)$ and angles $\left(^{\circ}\right)$ : $C(8)-C(1), 1.354(6) ; C(8)-C(9), 1.493(6) ; C(8)-C(14), 1.501(6) ; C(1)-$ $C(2), 1.494(6) ; C(1)-C(20), 1.484(6) ; C(23)-C(26), 1.455(6) ; C(26)-$ $C(27), 1.356(6) ; C(28)-N(1), 1.149(7) ; C(27)-C(29), 1.485(6) ; C(32)-$ $C(35), 1.475(6) ; C(40)-C(41), 1.484(7) ; C(41)-O(1), 1.187(7)$. $C(9)-C(8)-$ $C(14), 115.0(4) ; C(2)-C(1)-C(20), 115.3(4) ; C(26)-C(27)-C(29), 121.1(4) ;$ $C(27)-C(28)-N(1), 176.8(6) ; C(40)-C(41)-O(1), 125.8(6)$. were performed as shown in Fig. 2(A). As compared with PEG$\mathrm{AE}$, it can be observed from the spectrum of PEG-TPD1 that the characteristic $-\mathrm{CH}$ - proton peak belonging to the pyridine ring clearly appeared at $5.08 \mathrm{ppm}$ with the disappearance of characteristic-CHO peak at 10.06 ppm in TPDA dye, and some multiple peaks between 7.04 and 7.66 ppm were also found in the spectrum of PEG-TPD1 which were characteristic proton peaks of the aromatic ring of the introduced TPDA, indicating that dye TPDA was favourably incorporated into PEG-TPD polymers by Hantzsch reaction with functional monomer PEG-AE. The $-\mathrm{CH}_{2}-$ peaks linked to the ester groups of PEGMA appeared at $4.08 \mathrm{ppm}$. More importantly, the ${ }^{1} \mathrm{H}$ NMR spectrum of the polymer PEG-TPD2 obtained by the one-pot method was same with that of the polymers obtained by the two-step method, indicating that the structure of PEG-TPD2 was similar with that of PEG-TPD1 and the one-pot method was also successful.

These polymers were analyzed by GPC to study their $M_{\mathrm{n}}$ and PDI as shown in Fig. 2(B). The $M_{\mathrm{n}}$ of PEG-AE and PEG-TPD1 respectively were about 45000 and 52000 , indicating that in the second step, TPDA was favourably incorporated into the PEG-TPD1 polymer with PEG-AE polymer via the Hantzsch reaction. The $M_{\mathrm{n}}$ of the polymer PEG-TPD2 obviously decreased to 28000 as compared with PEG-TPD1, implying that the chains propagation was affected to some extent by Hantzsch reaction. According to the integral ratio of the characteristic peaks of $5.08 \mathrm{ppm}$ and $4.08 \mathrm{ppm}$, the molar fractions of TPDA in the polymers PEG-TPD1 and PEG-TPD2 was respectively about 9.5\% and $14.3 \%$ when the feeding ratio of TPDA was about $16.0 \%$, indicating that the Hantzsch reaction of one-pot strategy was more complete with the approximate disappearance of $-\mathrm{CH}_{2}-$ proton peak at $4.30 \mathrm{ppm}$ of PEG-AE as compared with the twosteps strategy. Further analysis on the effect of feeding ratio on polymer structure has also been carried out. When the feeding molar ratio of TPDA were respectively $20 \%$ and $25 \%$, the $M_{\mathrm{n}}$ of the polymers PEG-TPD3 and PEG-TPD4 respectively was about 31000 and 33000 and the molar fractions of TPDA were about $17.2 \%$ and $23.0 \%$. It can be seen that the $M_{\mathrm{n}}$ of the polymers gradually increases as the ratio of TPDA increasing for the same one-pot method, which was possibly as a result of the molecular weight difference of TPD-MA and PEGMA. Detailed

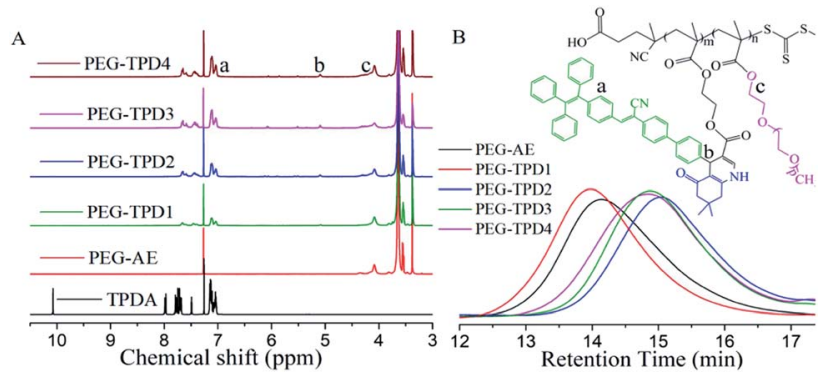

Fig. 2 (A) ${ }^{1} \mathrm{H}$ NMR spectra $\left(\mathrm{CDCl}_{3}\right)$ of the PEG-AE and PEG-TPD polymers. (B) The GPC traces (DMF) (PEG-AE, $M_{n}=45000, P D I=1.21$; PEG-TPD1, $M_{n}=52000, P D I=1.17 ;$ PEG-TPD2, $M_{n}=28000, P D I=$ 1.16; PEG-TPD3, $M_{n}=31000, P D I=1.16 ;$ PEG-TPD4, $M_{n}=33000$, $\mathrm{PDI}=1.19)$. 
investigation was further conducted to explore the Hantzsch reaction and RAFT polymerization process of TPDA, AEMA and PEGMA, and their kinetics procedure was demonstrated in Fig. S1. $\dagger$ The kinetics procedure showed a linear pseudo-firstorder kinetic with high monomer conversion. In the first stage about $7 \mathrm{~h}$, the rate of Hantzsch reaction was slightly higher than that of RAFT polymerization, however, it gradually decreased with the reaction time as compared with RAFT polymerization.

FT-IR spectra analysis was performed on TPDA, PEG-AE and PEG-TPD, and the results further confirmed the successful synthesis of PEG-TPD as shown in Fig. 3(A). For the TPDA dye, the characteristic absorption peaks of $-\mathrm{CHO},-\mathrm{CN}$ and aromatic ring obviously presented at 1690, 2210 and $3050 \mathrm{~cm}^{-1}$. Three typical peaks of the polymers PEG-AE were observed at 2870 , 1720 and $1100 \mathrm{~cm}^{-1}$, which should attribute to the stretching vibrations of $-\mathrm{CH}_{2}-, \mathrm{C}=\mathrm{O}$ and $\mathrm{C}-\mathrm{O}$, respectively. Compared with the PEG-AE, it can be seen that the polymers PEG-TPD have another absorption peak at $1640 \mathrm{~cm}^{-1}$ in addition to the above three typical absorption peaks, the intensity of which gradually enhance with the fraction increase of TPDA in PEG-TPD polymers, and this absorption peak can attribute to the stretching vibration of $\mathrm{C}=\mathrm{C}$ of TPDA. Moreover, the FT-IR spectra of polymers PEG-TPD by both one-pot and two-step methods show the same structure, which also indicated that TPDA in two methods successfully incorporated into polymers.

The above all results demonstrated the successful synthesis of PEG-TPD. The simultaneous presence of the hydrophobic TPDA segment and the hydrophilic PEGMA segment makes the fluorescent polymers PEG-TPD have amphiphilic property. In aqueous solution, the amphiphilic fluorescent PEG-TPD polymers will self-assemble into PEG-TPD FNPs, which will appear as a core-shell structure. The hydrophobic TPDA segment acted as the core, and the hydrophilic PEGMA segment covered the hydrophobic core as shell. The morphology and structure of the

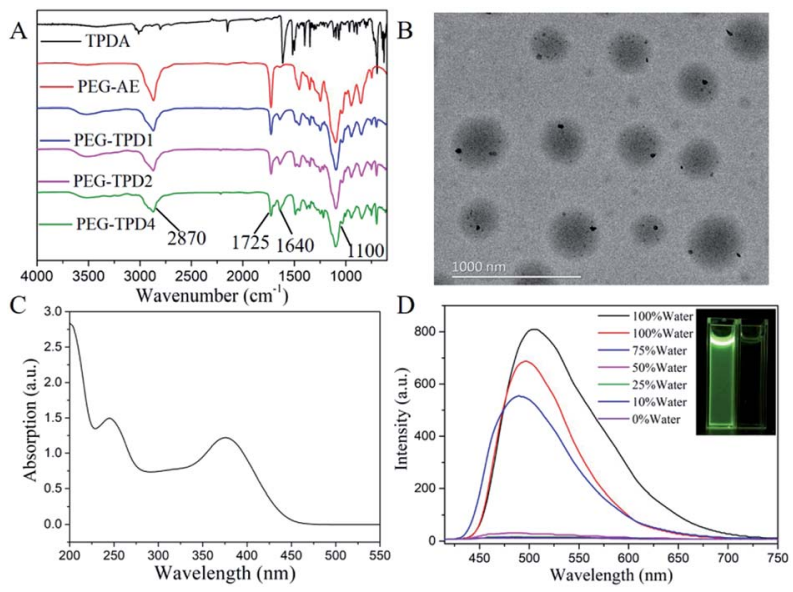

Fig. 3 Characterization of TPDA and PEG-TPD FPNs: (A) FT-IR spectra of TPDA, PEG-AE and PEG-TPD; (B) TEM image of PEG-TPD2 FPNs; (C) UV-visible absorption spectrum of PEG-TPD2 FPNs dispersed in water; (D) fluorescence emission spectra of PEG-TPD2 FPNs in water/THF mixed solutions with different volume ratios (left bottle (in pure water), right bottle (in pure THF)). nanoparticles were investigated by TEM, as shown in Fig. 3(B). A number of spherical particles with diameters of 200 to $300 \mathrm{~nm}$ have been observed, indicating that TPDA successfully combined with PEGMA to form amphiphilic fluorescent polymers PEG-TPD2 and they self-assembled into core-shell structure nanoparticles as expected in aqueous solution. Then the optical properties of PEG-TPD2 FPNs were further studied by UV-visible spectrophotometry and fluorescence spectrophotometry. As shown in Fig. 3(C), it can be clearly observed that there were two absorption peaks at $245 \mathrm{~nm}$ and $375 \mathrm{~nm}$. The absorption peak at $245 \mathrm{~nm}$ can be attributed to the $\pi \rightarrow \pi^{*}$ electrons transition because of the conjugate structure of PEGTPD2. The absorption peak at $375 \mathrm{~nm}$ should attribute to the $\mathrm{n}$ $\rightarrow \pi^{*}$ electrons transition due to the presence of the heteroatom $\mathrm{N}$ on the pyridine ring.

As shown in Fig. S2, $\uparrow$ from TPB to TPDA, the emission wavelength increased to $509 \mathrm{~nm}$ from $470 \mathrm{~nm}$ with stronger fluorescence intensity under the same condition because of the introduction of cyano groups and the increase of conjugation degree, which will reduce the energy difference between the first excitation state $S_{1}$ and the ground state $S_{0}$ and is very favorable for the biology imaging. In some organic solvents, the polymers PEG-TPD2 will have better solubility due to the presence of the hydrophobic TPDA segment, while in aqueous solution, they will self-assemble into nanoparticles. Fig. 3(D) shows the fluorescence emission spectra of PEG-TPD2 FPNs in water/THF mixed solutions with different volume ratios. There was a significant fluorescence emission peak at $509 \mathrm{~nm}$ in pure water solution, while there was almost no fluorescence in pure THF solution under the same conditions, which was consistent with the photograph taken under ultraviolet lamp shown in the illustration, in which PEG-TPD2 FPNs was dissolved separately in pure water (left) and pure THF (right). It was further observed that the smaller volume ratio of water in the mixed solution, the lower the fluorescence intensity, and when the volume ratio of water was lowered to $50 \%$, the fluorescence substantially disappeared, demonstrating that PEG-TPD2 had a typical AIE property. When the PEG-TPD2 polymers self-assembled into nanoparticles with core-shell structure in water, the hydrophobic TPDA segment aggregated in the interior of nanoparticles. Due to their distorted molecular conformation and the restricted intramolecular rotation, the non-radiative decay pathway was blocked, and then the excited state energy decayed through the radiation pathway which induced the fluorescence emission.

The above results indicate that PEG-TPD FPNs have good fluorescent performance and high water dispersity. In order to prove the potential application of PEG-TPD FPNs in the field of bioimaging, their biocompatibility was further evaluated. ${ }^{45-48}$ It can be clearly seen from the optical microscope images shown in Fig. S3 $\uparrow$ that the morphology of all cells treated with different concentrations of PEG-TPD2 FPNs did not change significantly and remained normal. Even though the concentrations of PEGTPD2 FPNs in the cell culture increased to a higher $80 \mu \mathrm{g} \mathrm{mL}^{-1}$, there was no significant change in cell morphology. Fig. 4(A) showed the results of CCK-8 test, in which L929 cells were incubated with PEG-TPD2 FPNs at different concentrations for 


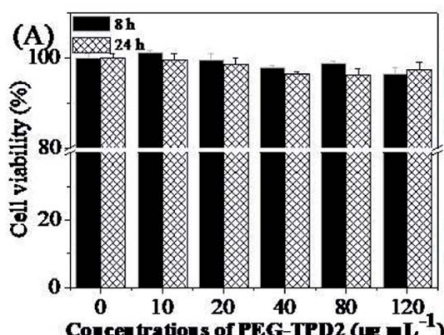

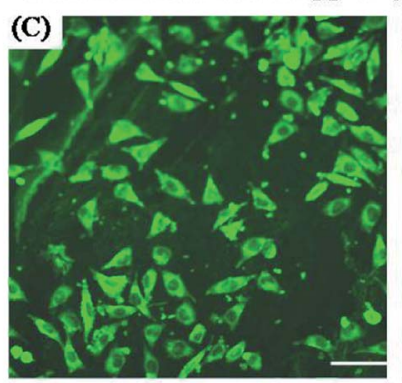

(B)
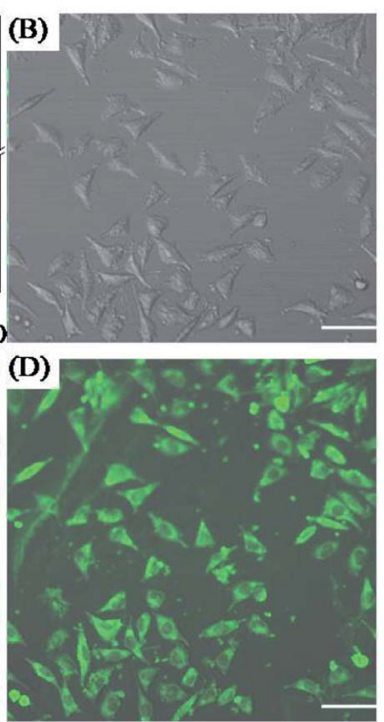

Fig. 4 Biocompatibility evaluations and cells imaging of PEG-TPD2 FPNs: (A) cell viability of PEG-TPD2 FPNs with L929 cells; (B)-(D) CLSM images of L929 cells incubated with $20 \mu \mathrm{g} \mathrm{mL}^{-1}$ of PEG-TPD2 FPNs, (B) bright field, (C) excited with a $405 \mathrm{~nm}$ laser, and (D) merged image of (B) and (C). Scale bar $=50 \mu \mathrm{m}$.

$8 \mathrm{~h}$ and $24 \mathrm{~h}$ to explore the cell viability of L929 cells. The results presented that the value of cell viability still exceeded $90 \%$ when the cells were incubated with different concentrations of PEGTPD2 FPNs even though the concentration was as high as 120 $\mu \mathrm{g} \mathrm{mL}^{-1}$, indicating that $\mathrm{L929}$ cells could maintain high viability. All the above results demonstrate that PEG-TPD2 FPNs have good biocompatibility and provides an attractive avenue for biological imaging applications.

PEG-TPD FPNs have the advantages of good fluorescence performance, high water dispersibility and good biocompatibility, which can be applied in biological imaging. Fig. 4(B)-(D) showed the uptake behavior of PEG-TPD2 FPNs by L929 cells from confocal laser scanning microscopy (CLSM). ${ }^{49,50}$ Before the test the L929 cells were incubated with PEG-TPD2 FPNs at an ideal concentration of $20 \mu \mathrm{g} \mathrm{mL} \mathrm{L}^{-1}$ for $3 \mathrm{~h}$ at $37^{\circ} \mathrm{C}$. As shown in Fig. 4(C), a strong green fluorescent signal was seen after the cells were irradiated with $405 \mathrm{~nm}$ laser. Further observation, the fluorescence in some areas was significantly weaker. Considering the size difference of cells, nucleus and PEG-TPD2 FPNs, it was considered that the regions where the fluorescence was significantly weaker were the nucleus. These results indicate that PEG-TPD2 FPNs can be easily absorbed by cells through endocytosis and dispersed in the cytoplasm after entering cells. Based on all the above tests, it can be concluded that PEG-TPD FPNs have significant advantages such as high fluorescence intensity, good water dispersibility, good biocompatibility, and easy absorption by cells, and can be attractively used in the field of biological imaging. In consideration of the many kinds of suitable monomers for RAFT polymerization and the high atomic economy of the Hantzsch reaction, their combination can incorporate special components into multifunctional polymers, which provides a promising approach for medical therapeutic diagnostics and bioimaging.

\section{Conclusions}

In summary, we synthesized a novel tetraphenylethenefunctionalized AIE-active dye TPDA. Compared with the reported dye TPB, the fluorescence intensity of TPDA is significantly enhanced with the obvious red shift of emission wavelength. Then the corresponding novel PEG-TPD polymers were successfully prepared by one-pot combination of RAFT polymerization and Hantzsch reaction. The structure of PEGTPD1 by the two-step process was similar with that of PEGTPD2 by the one-pot method at the same feeding ratio of TPDA and PEGMA. The $M_{\mathrm{n}}$ of PEG-TPD1 and PEG-TPD2 were respectively 52000 and 28000 , and the molar fractions of TPDA were respectively about $9.5 \%$ and $14.3 \%$. Subsequently, the effect of feeding ratio of TPDA and PEGMA on polymer structure was further studied, from which the $M_{\mathrm{n}}$ of the polymers gradually increases as the proportion of TPDA increasing. In aqueous solution, these amphiphilic PEG-TPD polymers tend to self-assemble into corresponding FPNs. The diameter of PEGTPD2 FPNs ranged from 200 to $300 \mathrm{~nm}$, and their fluorescence spectra have maximum emission peak at $509 \mathrm{~nm}$. The PEG-TPD FPNs have significant advantages of good fluorescence intensity, high water dispersibility and good biocompatibility, which can be attractively used in the field of bioimaging. Various AIE-based FPNs can also be prepared by using different AIE-active dyes through this convenient and effective one-pot combination of RAFT polymerization and Hantzsch reaction. In consideration of the many kinds of suitable monomers for RAFT polymerization and the high atomic economy of Hantzsch reaction, this one-pot method can incorporate some special components such as targeting drugs and imaging agents, etc. into multifunctional polymers, which provide a promising approach for medical therapeutic diagnostics and bioimaging.

\section{Conflicts of interest}

There are no conflicts to declare.

\section{Acknowledgements}

This research was supported by the Natural Science Foundation of Guangdong Province (2018A030313784), the National Science Foundation of China (No. 51673107, 21574073), and the Science and Technology Project of Zhongshan City of China (2018B1112).

\section{References}

1 M. Wang, G. Zhang, D. Zhang, D. Zhu and B. Tang, J. Mater. Chem., 2010, 20, 1858-1867.

2 Y. Liu, C. Deng, L. Tang, A. Qin, R. Hu, J. Sun and B. Tang, J. Am. Chem. Soc., 2011, 133, 660-663.

3 Y. Hong, L. Meng, S. Chen, C. Leung, L. Da, M. Faisal, D. Silva, J. Liu, J. Lam, X. Huang and B. Tang, J. Am. Chem. Soc., 2012, 134, 1680-1689.

4 J. Mei, Y. Hong, J. Lam, A. Qin, Y. Tang and B. Tang, Adv. Mater., 2014, 26, 5429-5479. 
5 E. Zhao, Y. Hong, S. Chen, C. Leung, C. Chan, R. Kwok, J. Lam and B. Tang, Adv. Healthcare Mater., 2014, 3, 88-96. 6 N. Rosi and C. Mirkin, Chem. Rev., 2005, 105, 1547-1562.

7 Q. Zhao, C. Huang and F. Li, Chem. Soc. Rev., 2011, 40, 25082524.

8 X. Zhang, S. Wang, C. Zhu, M. Liu, Y. Ji, L. Feng, L. Tao and Y. Wei, J. Colloid Interface Sci., 2013, 397, 39-44.

9 X. Zhang, J. Hui, B. Yang, Y. Yang, D. Fan, M. Liu, L. Tao and Y. Wei, Polym. Chem., 2013, 4, 4120-4125.

10 X. Michalet, F. Pinaud, L. Bentolila, J. Tsay, S. Doose, J. Li, G. Sundaresan, A. Wu, S. Gambhir and S. Weiss, Science, 2005, 307, 538-544.

11 R. Hu, N. Leung and B. Tang, Chem. Soc. Rev., 2014, 43, 44944562.

12 E. Ye, M. Regulacio, M. Bharathi, H. Pan, M. Lin, M. Bosman, K. Win, H. Ramanarayan, S. Zhang, X. Loh, Y. Zhang and M. Han, Nanoscale, 2016, 8, 543-552.

13 J. Luo, Z. Xie, J. Lam, L. Cheng, H. Chen, C. Qiu, H. Kwok, X. Zhan, Y. Liu, D. Zhu and B. Tang, Chem. Commun., 2001, 18, 1740-1741.

14 C. Zhang, X. Yao, J. Wang and X. Ma, Polym. Chem., 2017, 8, 4835-4841.

15 F. Gu, C. Zhang and X. Ma, Macromol. Rapid Commun., 2019, 40, 1800751.

16 S. Wang, F. Wang, C. Li, T. Li, D. Cao and X. Ma, Sci. China: Chem., 2018, 61, 1301-1306.

17 B. Sk and A. Patra, CrystEngComm, 2016, 18, 4290-4294.

18 Z. Huang, X. Zhang, X. Zhang, S. Wang, B. Yang, K. Wang, J. Yuan, L. Tao and Y. Wei, RSC Adv., 2015, 5, 89472-89477.

19 Z. Huang, X. Zhang, X. Zhang, C. Fu, K. Wang, J. Yuan, L. Tao and Y. Wei, Polym. Chem., 2015, 6, 607-612.

20 Z. Huang, X. Zhang, X. Zhang, B. Yang, Y. Zhang, K. Wang, J. Yuan, L. Tao and Y. Wei, Polym. Chem., 2015, 6, 2133-2138.

21 X. Zhang, M. Liu, B. Yang, X. Zhang and Y. Wei, Colloids Surf., B, 2013, 112, 81-86.

22 M. Liu, K. Wang, X. Zhang, X. Zhang, Z. Li, Q. Zhang, Z. Huang and Y. Wei, Tetrahedron, 2015, 71, 5452-5457.

23 X. Zhang, X. Zhang, B. Yang, J. Hui, M. Liu and Y. Wei, Colloids Surf., B, 2014, 116, 739-744.

24 R. Jiang, M. Liu, C. Li, Q. Huang, H. Huang, Q. Wan, Y. Wen, Q. Cao, X. Zhang and Y. Wei, Mater. Sci. Eng., C, 2017, 80, 708-714.

25 G. Feng, C. Tay, Q. Chui, R. Liu, N. Tomczak, J. Liu, B. Tang, D. Leong and B. Liu, Biomaterials, 2014, 35, 8669-8677.

26 Q. Zhao, K. Li, S. Chen, A. Qin, D. Ding, S. Zhang, Y. Liu, B. Liu, J. Sun and B. Tang, J. Mater. Chem., 2012, 22, 15128-15135.

27 D. Wang, J. Qian, W. Qin, A. Qin, B. Tang and S. He, Sci. Rep., 2014, 4, 4279.
28 J. Geng, K. Li, W. Qin, L. Ma, G. Gurzadyan, B. Tang and B. Liu, Small, 2013, 9, 2012-2019.

29 X. Zhang, X. Zhang, B. Yang, Y. Zhang, M. Liu, W. Liu, Y. Chen and Y. Wei, Colloids Surf., B, 2014, 113, 435-441.

30 W. Wu, C. Chen, Y. Tian, S. Jang, Y. Hong, Y. Liu, R. Hu, B. Tang, Y. Lee, C. Chen, W. Chen and A. Jen, Adv. Funct. Mater., 2010, 20, 1413-1423.

31 J. Chen, S. Luo, D. Xu, Y. Xue, H. Huang, Q. Wan, M. Liu, X. Zhang and Y. Wei, RSC Adv., 2016, 6, 54812-54819.

32 G. Zeng, M. Liu, R. Jiang, Q. Huang, L. Huang, Q. Wan, Y. Dai, Y. Wen, X. Zhang and Y. Wei, Polym. Chem., 2017, 8, 4746-4751.

33 Y. Cai, J. Fang, B. Wang, F. Zhang, G. Shao and Y. Liu, Sens. Actuators, B, 2019, 292, 156-163.

34 J. Wang and K. Matyjaszewski, J. Am. Chem. Soc., 1995, 117, 5614-5615.

35 G. Moad, E. Rizzardo and S. Thang, Aust. J. Chem., 2005, 58, 379-410.

36 R. Kakuchi, Angew. Chem., Int. Ed., 2014, 53, 46-48.

37 Z. Huang, Q. Chen, Q. Wan, K. Wang, J. Yuan, X. Zhang, L. Tao and Y. Wei, Polym. Chem., 2017, 8, 4805-4810.

38 H. Wu, L. Yang and L. Tao, Polym. Chem., 2017, 8, 5679-5687.

39 G. Liu, A. Shegiwal, Y. Zeng, Y. Wei, C. Boyer, D. Haddleton and L. Tao, ACS Macro Lett., 2018, 7, 1346-1352.

40 Z. Huang, R. Wang, Y. Chen, X. Liu, K. Wang, L. Mao, K. Wang, J. Yuan, X. Zhang, L. Tao and Y. Wei, Polym. Chem., 2019, 10, 2162-2169.

41 X. Zhang, Z. Chi, B. Xu, C. Chen, X. Zhou, Y. Zhang, S. Liu and J. Xu, J. Mater. Chem., 2012, 22, 18505-18513.

42 L. Tao, J. Liu and T. Davis, Biomacromolecules, 2009, 10, 2847-2851.

43 X. Li, C. Li, S. Wang, H. Dong, X. Ma and D. Cao, Dyes Pigm., 2017, 142, 481-490.

44 Z. Huang, K. Song, F. Liu, J. Long, H. Hu, H. Gao and Q. Wu, J. Polym. Sci., Part A: Polym. Chem., 2008, 46, 1618-1628.

45 X. Zhang, W. Hu, J. Li, L. Tao and Y. Wei, Toxicol. Res., 2012, 1, 62-68.

46 Y. Zhao, B. Yang, Y. Zhang, S. Wang, C. Fu, Y. Wei and L. Tao, Polym. Chem., 2014, 5, 6656-6661.

47 X. Zhang, H. Qi, S. Wang, L. Feng, Y. Ji, L. Tao, S. X. Li and Y. Wei, Toxicol. Res., 2012, 1, 201-205.

48 X. Zhang, S. Wang, M. Liu, J. Hui, B. Yang, L. Tao and Y. Wei, Toxicol. Res., 2013, 2, 335-342.

49 X. Zhang, S. Wang, C. Fu, L. Feng, Y. Ji, L. Tao, S. Li and Y. Wei, Polym. Chem., 2012, 3, 2716-2719.

50 B. Yang, Y. Zhang, X. Zhang, L. Tao, S. Li and Y. Wei, Polym. Chem., 2012, 3, 3235-3238. 